\title{
Task demands determine hand posture bias on conflict processing in a Simon task
}

\author{
Roman Liepelt $^{1} \cdot$ Rico Fischer $^{2}$
}

Published online: 15 July 2015

(C) Psychonomic Society, Inc. 2015

\begin{abstract}
A huge body of research in humans and monkeys has provided evidence for altered processing of items that are presented close to the hands. At the same time, the underlying mechanisms that explain why objects close to the hands are processed differently from objects far from the hands are still debated. Empirical demonstrations have provided evidence for the involvement of bottom-up influences, but also for top-down influences of task relevance. Objects close to the hands change spatial attentional processing or are subject to increased cognitive control. The present study demonstrated that variations in the task-processing demands predicted the hand posture influence on conflict resolution in a Simon task. Participants responded with their hands either at the monitor (close to the stimuli) or on their knees (far from the stimuli). The Simon effect was significantly reduced for the handsclose as compared to the hands-far condition when participants performed a numerical size judgment (Exps. 1 and 2). In contrast, the Simon effect was significantly increased for the hands-close condition when the Simon task consisted of a low-level perceptual feature discrimination (i.e., color task, Exp. 2). The obtained task-processing specificity provides further evidence that a highly flexible system underlies hand posture effects on stimulus processing.
\end{abstract}

Roman Liepelt and Rico Fischer contributed equally to this work.

Roman Liepelt

roman.liepelt@uni-muenster.de

Rico Fischer

rico.fischer@tu-dresden.de

1 Institute for Psychology, University of Muenster, 48149 Muenster, Germany

2 Department of Psychology, Technische Universität Dresden, 01062 Dresden, Germany
Keywords Simon effect $\cdot$ Cognitive control $\cdot$ Hand posture effect $\cdot$ Spatial attention $\cdot$ Stimulus-response compatibility

When people become interested in an object, they usually take the object in their hand, even when a direct manipulation of the object is not required. Objects close to our hands receive altered processing (Reed, Grubb, \& Steele, 2006), and visual detection rates improve for stimuli in the near-hand field (Schendel \& Robertson, 2004). Although a huge amount of research has compared behavioral task performance in various kinds of tasks between conditions in which stimuli were processed in hands-close and hands-far conditions (Abrams, Davoli, Du, Knapp, \& Paull, 2008; Davoli \& Abrams, 2009; Davoli \& Brockmole, 2012; Davoli, Brockmole, Du, \& Abrams, 2012; Davoli, Du, Montana, Garverick, \& Abrams, 2010; Reed, Betz, Garza, \& Roberts, 2010; Schultheis \& Carlson, 2013; Witt \& Proffitt, 2008), the underlying mechanisms for the observed hand posture bias on stimulus processing to date are still little understood. Most of the given research has shown that stimuli that are presented close to the hands benefit from enhanced visual analysis, in light of the assumption that objects that are close to the hands are possible candidates for action.

A popular assumption suggests that a change takes place in the spatial processing of (and/or the biased distribution of spatial attention to) the area near the hands (Abrams, Davoli, Du, Knapp, \& Paull, 2008; Davoli, Du, Montana, Garverick, \& Abrams, 2010; Reed et al., 2006; Schendel \& Robertson, 2004). It has been proposed, for example, that bimodal neurons show a spatially graded response pattern, with increasing firing rates the closer the stimulus is presented to the hands (cf. Graziano \& Gross, 1998; Reed et al., 2006). Consequently, the spatial proximity of a stimulus to the hand facilitates its detection (Reed et al., 2006). Further evidence that might be 
interpreted in terms of increased processing of spatial features has come from a visual attention allocation paradigm. In their inhibition of return (IOR) experiment, Abrams and colleagues showed no effect of hand proximity at short (300-ms) cuetarget intervals, but significant hand proximity effects at long (950-ms) cue-target intervals. They found that the magnitude of IOR was reduced in hands-close as compared to hands-far conditions. This effect suggests that hand position does not affect the ability to engage attention to the object at the cued location, but that hand nearness leads to delayed or disrupted disengagement of attention from the spatially cued object (Abrams et al., 2008).

In line with these findings, Wang, $\mathrm{Du}, \mathrm{He}$, and Zhang (2014) found facilitated low-level sensory processing mechanisms in a Simon (1990) task when the hands were close to the stimuli. In their experiment, participants responded with left or right buttonpresses to the identity (i.e., color) of stimuli presented unpredictably on either the left or the right of the screen. Although the location of stimulus presentation was irrelevant for the task, it is assumed to trigger automatic response activation of the spatially corresponding response hand. In classical dual-route models (e.g., Kornblum, Hasbroucq, \& Osman, 1990), this automatic response activation by direct-route processing either can facilitate response execution in compatible trials (e.g., a stimulus requiring a left response presented on the left) or can hinder response execution in incompatible trials (e.g., a stimulus requiring a left response presented on the right). Indirect-route processing is associated with rule-based S-R translation and intentional identification of the required response. Indirect-route processing also exhibits control over direct-route processing if automatic response activation by direct-route processing does not activate the correct response (Kornblum et al., 1990). The Simon effect consists in the difference between incompatible and compatible trials. Critically, Wang et al. obtained an increased Simon effect in the hands-close as compared to the hands-far condition. Thus, hands close to the lateralizedappearing stimuli enhanced spatial processing (or the processing of spatial features), and consequently increased automatic location-triggered response activation.

Findings such as these, however, stand in contrast to empirical observations that suggest an increased engagement of cognitive control for stimulus processing close to the hands that is not necessarily related to increased processing of a spatial dimension. In particular, recent studies have reported reduced interference effects in a nonspatial Stroop task (Davoli et al., 2010) and reduced task-switching costs for stimuli that appear close to the hands (Weidler \& Abrams, 2014). Because the stimuli used in these two studies were centrally presented color words (Davoli et al., 2010) or colored target shapes (Weidler \& Abrams, 2014), reduced interference cannot easily be explained by enhanced spatial processing. Reduced interference effects for hands-close stimuli do, therefore, suggest that stimuli close to the hands received increased levels of cognitive control (Weidler \& Abrams, 2014).

In line with this, Garza and colleagues (Garza, Strom, Wright, Roberts, \& Reed, 2013) manipulated task expectations by using the hands as visual anchors that were placed next to potential target locations, with a design that emphasized either the location of the hand relative to the target (i.e., the contralateral hand) or the location of the response hand itself. With the different designs, hand location speeded responses to targets appearing either close to the contralateral (nonresponding) hand or close to the response hand. Besides the typical bottom-up influence of attention to the visual field close to the response location, this study showed an effect of task expectations (i.e., top-down influence) that interacted with the hands' effects on visual attention.

Accumulating demonstrations of both bottom-up (e.g., Reed et al., 2006) and top-down (e.g., Garza et al., 2013) influences on hand posture effects suggest that a strict dichotomy of distinct mechanisms may be too simplistic to account for the differential processing of stimuli presented close to the hands. Instead, it appears to be important to determine the conditions under which hand position effects lead to enhanced visuospatial processing and/or increased cognitive control engagement. In fact, Reed and colleagues (Reed, Leland, Brekke, \& Hartley, 2013) recently applied event-related potentials (ERPs) and found different time courses for early bottom-up influences and later top-down influences. In particular, the Nd1 showed two distinct effects - an early facilitation of processing for all stimuli that appeared close to the hand versus a later-occurring P3 indicating cognitive control enhancement - that were modulated by hand position for targets, but not for nontargets.

It thus seems likely that stimulus- and task-specific cognitive processing might serve as predictors for different hand posture effects. On the one side, studies showing facilitating effects of hand proximity often require target detection and stimulus categorization on the basis of low-level perceptual features (e.g., Reed et al., 2006; Wang et al., 2014). In contrast, tasks that involved more complex (e.g., semantic) cognitive stimulus processing (e.g., Davoli et al., 2010; Weidler \& Abrams, 2014) have been associated with increased engagement of cognitive control for items appearing at a position close to the hand.

In the present study, we have built on this framework. In Simon tasks with low-level perceptual feature discriminations, the Simon effects increase in hands-close conditions, due to enhanced spatial processing (Wang et al., 2014). If increased cognitive processing demands were to emphasize an engagement of cognitive control for stimulus processing close to the hands, a Simon task should yield reduced effects - that is, the opposite finding to that reported by Wang et al. More specifically, any manipulation that exhibited increased engagement 
of cognitive control in the Simon task should predict reduced Simon effects due to enhanced target (indirect-route) processing - and thus, increased control over direct-route processing of the irrelevant spatial dimension (e.g., Stürmer, Leuthold, Soetens, Schröter, \& Sommer, 2002).

\section{Experiment 1}

To test this assumption, in Experiment 1 we implemented a version of the Simon task in which participants were not required to categorize low-level perceptual features (e.g., color) as in Wang et al. (2014), but to semantically categorize left- or right-presented digits as being either smaller or larger than 5 (Fischer, Dreisbach, \& Goschke, 2008; Fischer, Plessow, Dreisbach, \& Goschke, 2014). Thus, in order to correctly perform the task, the semantic size information needed to be accessed for each digit. As in the classical Simon task, the stimulus location triggered an automatic response activation process, resulting in the typical S-R conflict. Yet, in the number Simon task, the number stimuli also share a spatial leftright representation with increasing size information. This spatial-numerical association of response codes (SNARC effect; Dehaene, Bossini, \& Giraux, 1993) holds that righthand responses are faster for large number stimuli, and lefthand responses for small number stimuli, than are hand responses to stimuli of the opposite magnitudes. In the present number Simon task, only the compatible SNARC mappings were implemented (i.e., small digits-left response and large digits-right response).

Importantly, however, because of the spatial-numerical representations of numbers, the stimulus location might also conflict with the size information revealed by the identity of the digit. Therefore, the present paradigm contained an additional S-S conflict that might additively contribute to the usual S-R conflict of the Simon task. Note that a combination of multiple (e.g., S-S and S-R) conflict sources is not uncommon in typical conflict paradigms (e.g., the Stroop task). As a consequence, and important for the aim of the present study, the number Simon task involves much more complex cognitive processing of the stimuli than the low-level perceptual color discrimination in the study of Wang et al. (2014).

\section{Method}

Participants Twenty students of Technische Universität Dresden (11 male, nine female; $M_{\text {age }}=23.1$ years, $S D=3.70$ years) took part in a single experimental session and received course credits or $€ 3$ payment. All except one student claimed righthandedness.

Stimuli and apparatus The numbers 1 to 9 (except 5) served as the stimuli and were presented in white on a black background randomly $2.8 \mathrm{~cm}$ to the left or right of fixation (a + sign) on a 17-in. monitor. At a viewing distance of about $60 \mathrm{~cm}$, the numbers extended a visual angle of $0.48^{\circ}$ to $0.67^{\circ}$, with a horizontal spread of $2.7^{\circ}$ from fixation. Participants responded with the index finger of either hand. In the handsclose condition, a manual response key for each finger was located on the left and on the right side of the computer monitor and matched the height of stimulus presentation. In the hands-far condition, the response keys were attached to the left and right sides of a small cardboard box located on the knees. The distance between the response keys was matched between the hands-far and hands-close conditions.

Stimulus presentation and data recording were controlled by the Presentation software (version 16.5; Neurobehavioral Systems, Inc., Albany, CA, USA).

Procedure We used a number version of the Simon task (Fischer et al., 2008; Fischer et al., 2014), in which participants categorized digits (1-4 and 6-9) as being smaller or larger than 5 with left-hand (smaller) and right-hand (larger) keypresses. The lateralized presentation of the stimuli produced a response conflict (i.e., incompatible trial) when the spatially assigned response (e.g., the right key) to the identity of the stimulus (e.g., the digit 9) did not match the location of the stimulus location on the display (e.g., the left location). In case of compatible trials, the required response to the identity of the stimulus (e.g., right key to the digit 9) was spatially compatible to the location of stimulus presentation on the screen (e.g., the right side). The performance in compatible trials subtracted from that in incompatible trials denoted the Simon effect.

Each trial started with a fixation sign for $1,000 \mathrm{~ms}$, after which a target stimulus was added for $200 \mathrm{~ms}$. The trial ended when a response was delivered or a maximum of 1,600 ms; the fixation sign remained on the screen for the entire trial. For correct trials, feedback was realized by a blank screen for $300 \mathrm{~ms}$. For misses or erroneous responses, the feedback $z u$ langsam ("too slow") or falsch ("false") was presented, respectively. A random interval with a blank screen between 100 and 1,000 ms was implemented before the next trial started.

The experiment consisted of two hand positions that were realized in separate blocks, containing 64 compatible and 64 incompatible trials each. Within each block of 128 trials, each combination of 8 stimuli (1-9, except 5$) \times 2$ locations (left vs right) was presented eight times. A short break was provided after 64 trials. The block order was counterbalanced between participants, and each block started with a brief practice of 16 trials.

Design A 2 (Simon Compatibility) $\times 2$ (Hand Position) repeated measures design was applied. 


\section{Results and discussion}

For the response time (RT) analysis only, error trials (5.1\%) and trials that were above or below $2.5 S D$ s of the individual condition mean $(2.7 \%)$ were removed prior to the analysis.

A repeated measures analysis of variance (ANOVA) revealed that responses were clearly affected by Simon compatibility, $F(1,19)=23.54, p<.001, \eta^{2}=.55$, with faster responses in compatible ( $433 \mathrm{~ms}$ ) than in incompatible (453 ms) trials. Hand position, on the other hand, did not affect response speed, $F<1$. Most importantly, the factors Hand Position and Simon Compatibility interacted, $F(1,19)=6.18, p=.022, \eta^{2}$ $=.25$. That is, the Simon effect was only half the size when hands were located close to the stimuli $(13.6 \mathrm{~ms})$ as when they were located far from the stimuli $(26.1 \mathrm{~ms})$ on the screen (see Fig. 1).

For error rates, a significant effect of Simon compatibility was observed, $F(1,19)=9.59, p=.006, \eta^{2}=.34$ : More errors were committed for incompatible (6.4\%) than for compatible $(3.8 \%)$ trials. We observed no difference in error rates between hand positions, $F<1$. The Simon effects in error rates for the hands-close and hands-far conditions amounted to $2.3 \%$ and $3.0 \%$, respectively (see Fig. 1). This difference, however, was not significant, $F<1$.

In line with the prediction that complex cognitive stimulus processing would enhance cognitive control engagement in close hand space, we found reduced Simon effects for a Simon task that was based on number size judgment.

\section{Experiment 2}

The reduced Simon effect in Experiment 1 stands in contrast to the finding of an increased Simon effect in hands-close conditions reported by Wang et al. (2014). We reasoned that an increased demand for cognitive stimulus processing would lead to greater engagement of cognitive control over low-level sensory processing mechanisms in the hands-close condition. Although the findings of Experiment 1 are straightforward, in Experiment 2 we aimed to substantiate our argumentation by (1) replicating the findings of Experiment 1 in an independent sample and (2) demonstrating the differential effects of hand posture on the Simon effect in a single within-subjects design with varying task-processing demands.

In the Simon task of Experiment 2, bivalent stimuli were implemented. That is, number stimuli were presented lateralized and randomly in either red or green. In one part of the experiment, participants were instructed to categorize numbers as being either smaller or larger than 5 , and to ignore the color information. In another part, participants were told to perform a color discrimination and to ignore the number information. By instructing participants how to respond to bivalent number stimuli (i.e., a number categorization Simon task

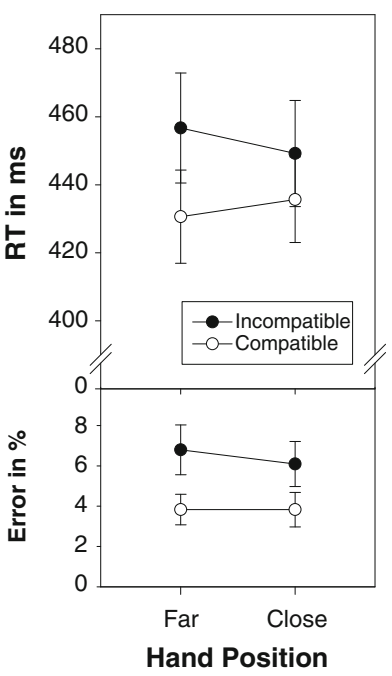

Fig. 1 Response times (RTs, in milliseconds) and percent errors of Exp. 1 for Simon compatible and incompatible trials, for conditions with the hands located either far from stimuli (on the knees) or close to stimuli (at the monitor), respectively. Error bars represent standard errors of the means

or a color Simon task), we expected enhanced cognitive control engagement and, thus, reduced Simon effects in close hand space when numbers had to be categorized according to their numerical size, and the reverse pattern of enhanced visuospatial processing (i.e., a greater Simon effect) when participants were asked to categorize a pure perceptual feature such as color. This account of hand bias effects on attention assumes contributions of both bottom-up and top-down influences, depending on the type of task the hand position manipulation was applied to.

\section{Method}

Participants Fifty-six students of Technische Universität Dresden (46 female, 10 male; $M_{\text {age }}=25.93$ years, $S D=$ 4.38 years) took part in a single experimental session and received course credits or $€ 5$ payment. All students claimed right-handedness.

Stimuli and apparatus The stimuli and apparatus were identical to those of Experiment 1, except that bivalent stimuli were created by presenting all number stimuli in either green (HSB: 85, 231, 150) or red (HSB 0, 231, 150) color.

Procedure Prior to the experiment, participants performed a color vision test consisting of the first ten plates of Ishihara's Test for Colour Blindness (http://virtualcampus.kcl.ac.uk/ colourblindness/).

In the number task, participants were instructed to ignore the stimulus color and to categorize the number stimuli as being smaller or larger than 5 . The stimulus-response assignments were identical to those in Experiment 1. In the color task, participants were instructed to ignore the identity of the 
numbers and to categorize the number stimuli according to color. The same response key setup was used. However, half of the participants received red-left and green-right mappings, whereas the other half received green-left and red-right mappings. The timing of trial events was the same as in Experiment 1 .

As in Experiment 1, the number task included four blocks of 64 trials, two for each hand condition (close vs. far), amounting to a total of 256 trials. The same trial structure was applied for the color task. Together, each participant performed a total of 512 trials. The order of tasks (number first vs. color first) and the order of hand positions (far first vs. close first) were counterbalanced between participants. For a single participant, however, the order of hand positions did not change from the color to the number task, or vice versa. Each change of hand position was accompanied by 16 trials of familiarization.

Design A 2 (Task) $\times 2$ (Simon Compatibility) $\times 2$ (Hand Position) repeated measures design was applied.

\section{Results and discussion}

All participants answered correctly to the ten plates of the color test. One participant was excluded from the analyses due to overall high error rates of $11.1 \%$ (more than 2.5 SDs from the sample mean). For the RT analysis only, error trials $(4.86 \%)$ and trials that were more than $2.5 S D$ s above or below the individual condition mean $(2.60 \%)$ were removed prior to the analysis. Because of the expected small effect sizes, and because the change of task sets with bivalent stimuli might induce additional noise in the data, we treated the first 64 trials after each condition change as training and restricted the analyses to the second block of each hand condition. This led to a cell mean of 32 trials. $^{1}$

The repeated measures ANOVA revealed a main effect of task, $F(1,54)=17.85, p<.001, \eta^{2}=.25$, and a main effect of Simon compatibility, $F(1,54)=76.27, p<.001, \eta^{2}=.59$. Responses were faster in the color task $(423 \mathrm{~ms})$ than in the number task (449 ms), and were generally faster for compatible (424 ms) than for incompatible (448 ms) trials. The Simon effects, however, did not differ between the tasks, $F(1,54)=$ $1.50, p=.226, \eta^{2}=.03$, nor did hand position influence the RTs, $F(1,54)=1.01, p=.319, \eta^{2}=.02$. Most importantly, and as predicted, the effect of hand position on Simon compatibility was task specific, $F(1,54)=4.76, p=.033, \eta^{2}=.08$, showing opposite patterns for the number and color tasks (see Fig. 2). For the number task, we replicated the findings

\footnotetext{
${ }^{1}$ Using the entire data set, the analyses below demonstrated that the important three-way Task $\times$ Simon Compatibility $\times$ Hand Position interaction was still significant for error rates, $F(1,54)=5.33, p=$ $.025, \eta^{2}=.09$, and approached significance for RTs, $F(1,54)=3.91$, $p=.053, \eta^{2}=.07$
}

of Experiment 1. That is, the Simon effect grew smaller when hands were positioned close to the stimuli, $F(1,54)=4.14, p=$ $.047, \eta^{2}=.07$. For the color task, this effect was slightly reversed, but failed to achieve statistical significance, in RTs, $F(1,54)=1.13, p=.293, \eta^{2}=.02$.

For error rates, a strong effect of Simon compatibility was observed, $F(1,54)=57.29, p<.001, \eta^{2}=.52$ : More errors were produced in incompatible $(6.38 \%)$ than in compatible $(3.61 \%)$ trials. Slightly more errors were committed in the color task $(5.47 \%)$ than in the number task $(4.52 \%), F(1$, $54)=5.14, p=.027, \eta^{2}=.09$. Most importantly, the significant three-way interaction, $F(1,54)=6.04, p=.017, \eta^{2}=.10$, mirrored the one in the RT data and confirmed the reverse influences of hand position on Simon effects in the number and color tasks (see Fig. 2). In the number task, the Simon effect was marginally smaller when hands were close to the stimuli $(2.41 \%)$ than when they were far away $(3.52 \%)$. This difference, however, was not significant, $F(1,54)=0.74, p=$ $.395, \eta^{2}=.01$. In the color task, however, the Simon effect was larger when hands were close to the stimuli $(4.03 \%)$ than when hands were far from the stimuli $(0.91 \%), F(1,54)=$ $6.78, p=.012, \eta^{2}=.11$.

Experiment 2 provided a reversed result pattern of the hand position influence on the Simon effect for the size versus color judgment, thus confirming the hypothesis of task-specificity. Replicating findings from Experiment 1, smaller Simon effects were found in the hands-close than in the hands-far condition when participants categorized numbers according to their numerical size. In contrast, evidence for increased Simon effects were found in the hands-close relative to the hands-far condition when participants responded to the color of the stimuli, thus replicating previous observations reported by Wang et al. (2014).

Evidence for the opposite effect pattern was obtained in the form of significant three-way interactions for both dependent measures (RTs and error rates). It should be noted, however, that subsequent separate testing revealed statistical proof only in a single measure - that is, in RTs for number judgments and errors for color judgments - whereas the complementary measure was only numerically confirmed. We think that the use of bivalent stimuli may have supported information processing of the irrelevant stimulus feature at least to some degree, and may thus have led to weakened clearness of the results. In any case, Experiment 2 provided clear evidence for an opposite influence of hand proximity on the Simon effect, depending on the instructed task processing.

\section{General discussion}

Previous research has provided evidence for altered processing of items that appear close to the hands, indicating the involvement of bottom-up and/or top-down influences of task 


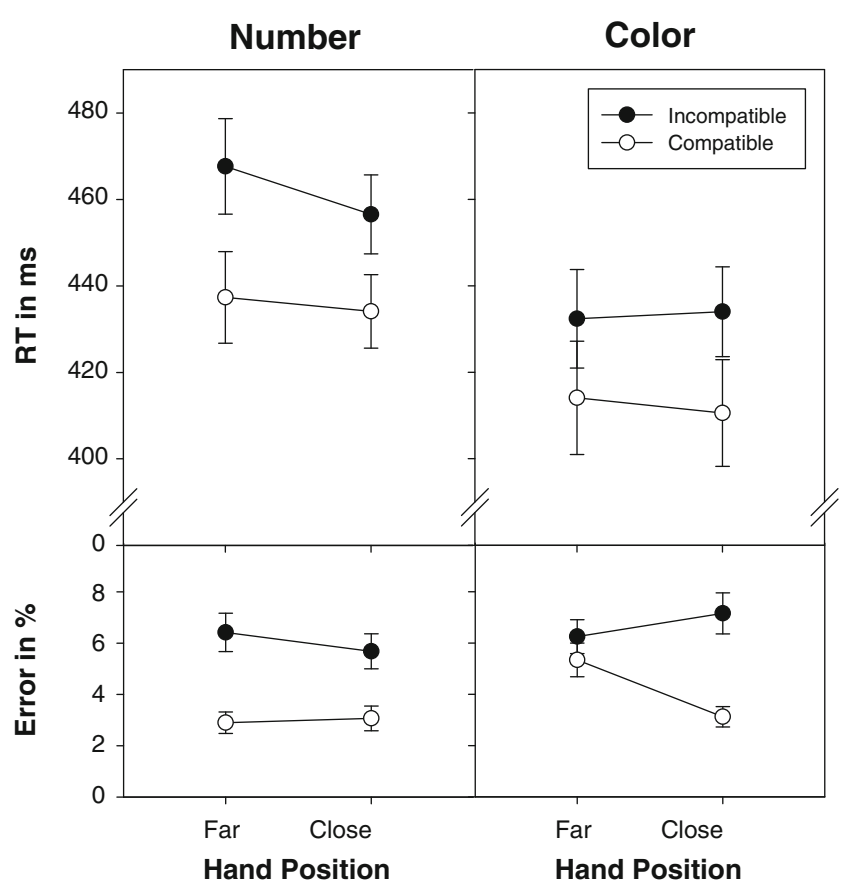

Fig. 2 Response times (RTs, in milliseconds) and percent errors of Exp. 2 for Simon compatible and incompatible trials, for conditions with the hands located either far from stimuli (on the knees) or close to stimuli (at the monitor), respectively, in (Left) the Simon number task and (Right) the Simon color task. Error bars represent standard errors of the means

relevance. In the present study, we aimed to test whether variations in task-processing demands predict the hand posture influence on conflict resolution in a Simon task. In a first experiment, we applied a hand position manipulation to a Simon task in which participants had to semantically categorize digits as being either smaller or larger than 5 (Fischer et al., 2008; Fischer et al., 2014). The results showed a significantly smaller Simon effect when the hands of the participant were located close to the stimuli than when they were located far from the stimuli. Reduced Simon effects are in line with the assumption that people exhibit increased cognitive control for items that appear close to the hands (Weidler \& Abrams, 2014) by enhancing the processing of the task-relevant information, thus reducing the impact of spatial location in triggering automatic response activation. The finding of a smaller Simon effect for stimuli close to the hands is in line with previous results showing reduced interference in a Stroop task (Davoli et al., 2010) and reduced task-switching costs (Weidler \& Abrams, 2014) for stimuli appearing in close hand space. It should be noted that in selective attention tasks, such as the Stroop or Simon tasks, increased cognitive control can act to inhibit task-irrelevant stimulus features (e.g., location information) and/or specifically to strengthen task-relevant processing (e.g., stimulus identity). Here we assume that the underlying mechanism of cognitive control might be related to a heightened activation level of the relevant task representations (e.g., Egner \& Hirsch, 2005). This reasoning incorporates previous findings of less efficient search rates in visual search and reduced secondary-target detection rates in attentional blink tasks (Abrams et al., 2008) when stimuli are close to the hands. A more thorough processing of the currently relevant stimulus features reduces the impact of task-irrelevant stimulus features at the cost of slower attentional shifts between currently processed and new items (i.e., disengagement costs). With respect to the Simon task that we applied, hand proximity seems to facilitate indirect route processing (Kornblum et al., 1990) over the processing of the automatic spatial route, and thus benefits the activation of the correct stimulus-response translation rule (Weidler \& Abrams, 2014).

In our second experiment, we used bivalent stimuli (i.e., the number stimuli from Exp. 1 presented in green or red). When participants had to respond to the number information (i.e., ignoring the color), we replicated our finding from Experiment 1 of a reduced Simon effect for hands-close conditions as compared to hands-far conditions. When participants were instructed to respond to the stimulus color (i.e., ignoring the number information), we found the reverse pattern. The Simon effect was increased when stimuli were presented close to as compared to far from the hands, replicating the findings of Wang et al. (2014). That a manipulation of task-processing demands (i.e., low-level perceptual feature discrimination vs. semantic number size judgment) can reverse the hand bias effect not only suggests that hand position effects are driven by bottom-up information, but support the assumption of topdown mediation of hand position effects on attention (Garza et al., 2013; Reed et al., 2013). The finding of the observed reversal of the hand bias effect by the task instructions is in line with the previous work of Davoli and Abrams (2009), showing that merely instructing participants to imagine holding their hands on the monitor led to a perceptual enhancement of the items in close hand space.

Although more facilitatory hand position effects tend to occur for less demanding target detection tasks (e.g., Reed et al., 2013), more inhibitory cognitive control effects are found for more cognitively demanding tasks requiring object recognition (Davoli et al., 2010) or semantic target categorization, as in our study. It seems as if hand position effects are mediated by a flexible system whereby bottom-up sensoryrelated inputs and top-down sources of control compete for attentional resources, directing attention to particular objects and/or spatial information (Garza et al., 2013; Kastner \& Ungerleider, 2000; Reed et al., 2013). In our cognitively more demanding categorical number Simon task, the cognitive complexity seems to be predominant, whereas in the color Simon task of Wang et al. (2014) and our Experiment 2, low-level sensory-related inputs prevail. Hand bias effects for cognitively demanding tasks, which in our case was a Simon task with multiple (e.g., S-S and S-R) conflicts, seem to enhance attention to the task-relevant stimulus information. 
In the Simon task, strengthening the processing of taskrelevant information along the indirect route also strengthens the impact of the indirect route (Kornblum et al., 1990), exerting control over direct-route processing when the wrong response is activated, thus decreasing the Simon effect for the hands-close conditions. The idea that we propose for the hand space bias, that a flexible weighting of bottom-up versus topdown signals for attention depends on the task demands, is in line with the previously suggested trade-off between spatial and semantic processing in hands-close conditions (Davoli et al., 2010). The dissociation of bottom-up versus top-down attention signals for hand position effects is supported by the recent ERP work of Reed and colleagues (2013). They found an early and less selective hand bias effect on the Nd1, probably reflecting early processes of multisensory integration (Taylor-Clarke, Kennett, \& Haggard, 2002), and on the later P3 component, which was selective for target stimuli (Polich, 2007; Reed et al., 2013). The observed target selectivity of the later P3 component is in line with the idea of enhanced target processing that we proposed as the underlying mechanism for the hand bias effect for cognitively demanding tasks. Our findings suggest that the effects of body information on visual processing depend on the task demands and on the relative weighting of cognitive control over low-level sensory processing mechanisms. Hand bias effects seem to be related to changes in early sensory processing based on bimodal neurons (Reed et al., 2006) and later top-down attentional control biases related to the perceived goal of the task (Garza et al., 2013), as well as to more anterior cognitive control networks (Botvinick, Cohen, \& Carter, 2004).

Taken together, the present study provides a new test for the potential cognitive mechanisms underlying altered processing of stimuli that are presented close to the hands. Whether hand bias effects lead to a performance improvement or impairment seems to critically depend on the task demands. We found a decreased Simon effect when hands were located close to rather than far from the stimuli in a semantic number categorization Simon task, but the reverse effect (i.e., increased Simon effect for hands-close conditions) for lowlevel perceptual feature discrimination in a color Simon task. These findings are in line with the assumption that hand bias effects are the product of the attentional competition of bottom-up inputs and top-down control for attentional resources (Garza et al., 2013; Reed et al., 2013), demonstrating the flexibility of the system underlying hand bias effects.

Furthering the understanding of how processing benefits (e.g., reduced distraction) and costs (e.g., delayed attentional disengagement) in close hand space arise will be informative not only in basic cognitive science, but also for the development of real-life applications, such as visual-manual display interaction systems. For tasks in which increased levels of cognitive control are advantageous, new displays allowing direct visual-manual interaction could help to optimize task performance. In this respect, it is important to demonstrate when stimuli presented close to the hands are more subject to heightened control, and are thus less susceptible to distracting and potentially error-prone influences, and when they lead to enhanced bottom-up sensory effects. Future research should test whether frontal cognitive control networks (Ridderinkhof, Ullsperger, Crone, \& Nieuwenhuis, 2004) in fact interact with the neurons coding multisensory information in a hand-centered space in the parietal cortex (Graziano \& Gross, 1995; Makin et al., 2007; Reed et al., 2006) in order to flexibly exert an appropriate level of cognitive control by hand proximity, which is a clear prediction that can be drawn from our findings.

Author note We thank Marcel Kurtz for assistance in data collection. This research was partly supported by the German Research Foundation, Grant Numbers DFG LI 2115/1-1 and 1-3, awarded to R.L., and DFG SFB 940/1, Project A3, awarded to R.F. We thank Richard A. Abrams and one anonymous reviewer for their helpful comments on an earlier version of this article.

\section{References}

Abrams, R. A., Davoli, C. C., Du, F., Knapp, W. K., III, \& Paull, D. (2008). Altered vision near the hands. Cognition, 107, 1035-1047. doi:10.1016/j.cognition.2007.09.006

Botvinick, M. M., Cohen, J. D., \& Carter, C. S. (2004). Conflict monitoring and anterior cingulate cortex: An update. Trends in Cognitive Sciences, 8, 539-546. doi:10.1016/j.tics.2004.10.003

Davoli, C. C., \& Abrams, R. A. (2009). Reaching out with the imagination. Psychological Science, 20, 293-295. doi:10.1111/j.1467-9280. 2009.02293.x

Davoli, C. C., \& Brockmole, J. R. (2012). The hands shield attention from visual interference. Attention, Perception, \& Psychophysics, 74, 1386-1390. doi:10.3758/s13414-012-0351-7

Davoli, C. C., Brockmole, J., Du, F., \& Abrams, R. A. (2012). Switching between global and local scopes of attention is resisted near the hands. Visual Cognition, 20, 659-668. doi:10.1080/13506285. 2012.683049

Davoli, C. C., Du, F., Montana, J., Garverick, S., \& Abrams, R. A. (2010). When meaning matters, look but don't touch: The effects of posture on reading. Memory \& Cognition, 38, 555-562. doi:10.3758/MC. 38.5.555

Dehaene, S., Bossini, S., \& Giraux, P. (1993). The mental representation of parity and number magnitude. Journal of Experimental Psychology: General, 122, 371-396. doi:10.1037/0096-3445.122. 3.371

Egner, T., \& Hirsch, J. (2005). Cognitive control mechanisms resolve conflict through cortical amplification of task-relevant information. Nature Neuroscience, 8, 1784-1790. doi:10.1038/nn1594

Fischer, R., Dreisbach, G., \& Goschke, T. (2008). Context-sensitive adjustments of cognitive control: Conflict-adaptation effects are modulated by processing demands of the ongoing task. Journal of Experimental Psychology: Learning, Memory, and Cognition, 34, $712-718$.

Fischer, R., Plessow, F., Dreisbach, G., \& Goschke, T. (2014). Individual differences in the context-dependent recruitment of cognitive control: Evidence from action versus state orientation. Journal of Personality. doi:10.1111/jopy.12140 
Garza, J. P., Strom, M. J., Wright, C. E., Roberts, R. J., Jr., \& Reed, C. L. (2013). Top-down influences mediate hand bias in spatial attention. Attention, Perception, \& Psychophysics, 75, 819-823. doi:10.3758/ s13414-013-0480-7

Graziano, M. S. A., \& Gross, C. G. (1995). From eye to hand. In J. King \& K. H. Pribram (Eds.), Scale in conscious experience: Is the brain too important to be left to specialists to study? (pp. 117-129). Mahwah, NJ: Erlbaum.

Graziano, M. S. A., \& Gross, C. G. (1998). Spatial maps for the control of movement. Current Opinion in Neurobiology, 8, 195-201. doi:10. 1016/S0959-4388(98)80140-2

Kastner, S., \& Ungerleider, L. (2000). Mechanisms of visual attention in the human cortex. Annual Review of Neuroscience, 23, 315-341. doi:10.1146/annurev.neuro.23.1.315

Kornblum, S., Hasbroucq, T., \& Osman, A. (1990). Dimensional overlap: Cognitive basis for stimulus-response compatibility-A model and taxonomy. Psychological Review, 97, 253-270. doi:10.1037/0033295X.97.2.253

Makin, T. R., Holmes, N. P., \& Zohary, E. (2007). Is that near my hand? Multisensory representation of peripersonal space in human intraparietal sulcus. Journal of Neuroscience, 27, 731-740.

Polich, J. (2007). Updating P300: An integrative theory of P3a and P3b. Clinical Neurophysiology, 118, 2128-2148.

Reed, C. L., Betz, R., Garza, J. P., \& Roberts, R. J., Jr. (2010). Grab it! Biased attention in functional hand and tool space. Attention, Perception, \& Psychophysics, 72, 236-245. doi:10.3758/APP.72.1. 236

Reed, C. L., Grubb, J. D., \& Steele, C. (2006). Hands up: Attentional prioritization of space near the hand. Journal of Experimental Psychology: Human Perception and Performance, 32, 166-177. doi:10.1037/0096-1523.32.1.166

Reed, C. L., Leland, D. S., Brekke, B., \& Hartley, A. A. (2013). Attention's grasp: Early and late hand proximity effects on visual evoked potentials. Frontiers in Psychology, 4, 420. doi:10.3389/ fpsyg.2013.00420

Ridderinkhof, K. R., Ullsperger, M., Crone, E. A., \& Nieuwenhuis, S. (2004). The role of the medial frontal cortex in cognitive control. Science, 306, 443-447. doi:10.1126/science.1100301

Schendel, K., \& Robertson, L. C. (2004). Reaching out to see: Arm position can attenuate human visual loss. Journal of Cognitive Neuroscience, 16, 935-943.

Schultheis, H., \& Carlson, L. A. (2013). Determinants of attentional modulation near the hands. Frontiers in Psychology, 4, 858. doi: 10.3389/fpsyg.2013.00858

Simon, J. R. (1990). The effects of an irrelevant directional cue on human information processing. In R. W. Proctor \& T. G. Reeve (Eds.), Stimulus-response compatibility (pp. 31-86). Amsterdam, The Netherlands: Elsevier.

Stürmer, B., Leuthold, H., Soetens, E., Schröter, H., \& Sommer, W. (2002). Control over location-based response activation in the Simon task: Behavioral and electrophysiological evidence. Journal of Experimental Psychology: Human Perception and Performance, 28, 1345-1363. doi:10.1037/0096-1523.28.6.1345

Taylor-Clarke, M., Kennett, S., \& Haggard, P. (2002). Vision modulates somatosensory cortical processing. Current Biology, 12, 233-236.

Wang, X., Du, F., He, X., \& Zhang, K. (2014). Enhanced spatial stimulus-response mapping near the hands: The Simon effect is modulated by hand-stimulus proximity. Journal of Experimental Psychology: Human Perception and Performance, 40, 2252-2265. doi:10.1037/a0038140

Weidler, B. J., \& Abrams, R. A. (2014). Enhanced cognitive control near the hands. Psychonomic Bulletin \& Review, 21, 462-469.

Witt, J. K., \& Proffitt, D. R. (2008). Action-specific influences on distance perception: A role for motor simulation. Journal of Experimental Psychology: Human Perception and Performance, 34, 1479-1492. doi:10.1037/a0010781 